\title{
VERORDNUNGEN UND STEREOTYPE DEUTSCHE UND POLNISCHE WIRTSHÄUSER IN DER FRÜHEN NEUZEIT
}

Iß was gar is, trinck was klar is, sag was war is.

Martin Luther

Die frühneuzeitliche Metropole Danzig übte auf Reisende eine große Anziehungskraft aus. Sie war ein Sinnbild des Bekannten, des Vertrauten. Die Fremden bewunderten die prachtvolle Architektur, das Zeughaus, die Kirchen, die Festung Weichselmünde. Reisende, die sich von Westen, aus Berlin oder Hamburg, Lübeck, Bremen Richtung Danzig, Königsberg bis nach Riga auf dem Landwege begaben, wählten verständlicherweise die nördliche Route, durch Frankfurt, nach bzw. um Stettin, Köslin, Stolp, weiter in die Kaschubei. Von Danzig aus fuhren sie weiter gen Osten nach Königsberg oder längs der Weichsel über Thorn nach Warschau. Die Nachbarschaft Preußens zu Polen prägte auf natürliche Weise das Schicksal beider Staaten für Jahrhunderte, ob es Kurbrandenburg, Herzogtum Preußen, Königlich Preußen unter der polnischen Krone, Königreich Preußen oder Ost- und Westpreußen hieß. Die aus Berlin an die Ostsee führenden Straßen waren mit denen Richtung Frankreich und Italien nicht zu vergleichen, aber sie stellten mit der hansischen Vergangenheit im Hintergrund eine wichtige Ader für den Handel, die Postverbindungen und den alltäglichen Bedarf der Bewohner und der reisenden Fremden auf der Ost-West-Achse dar. Diejenigen, die sich auf diese Reise vorbereiten wollten, studierten Wegweiser oder Reyßbüchlein von Autoren wie Johann Heinrich Seyfried, Hierony- 
mus Uhesel vel Vhessel, Daniel Wintzenberger, Peter Ambrosius Lehmann ${ }^{1}$. Wenn man sich vergegenwärtigt, dass manche dieser Reiseführer an der Schwelle des 17. und 18. Jahrhunderts bis zu 17 Auflagen erreichten, so bekommen wir ein Bild von der Popularität dieser kleinen Druckwerke und von einem Bedarf auf dem Buchmarkt, den die Buchdrucker und -händler nicht ignorieren konnten. Mehr noch, die Verleger, die die Attraktivität ihrer anderen Produkte erhöhen wollten, gaben Wegweiser als Beilage zu ihren eigentlichen Periodika heraus wie Georg Greflinger, Redakteur des Nordischen Mercurius ${ }^{2}$. In seinem Wegweiser von 1674 beschrieb er auch die Route durch Preußen ab Stettin bis nach Danzig, Elbing und Thorn.

Die in den Reiseführern genannten Wirtshäuser, Gasthäuser, Dorfkrüge erwarben sich einen sehr positiven oder eher einen negativen Ruhm. Ihre Namen wurden in den Reiseführern ihrer Epoche genannt, sie wurden empfohlen oder eben nicht. So wurde dem Reisenden abgeraten, bestimmte Gasthäuser zu besuchen und geraten, eher einen Umweg um sie herum zu machen. Für einen Reisenden war oft von entscheidender Bedeutung, ob er und seine Pferde dort eine sichere Unterkunft bekommen konnten. Gerade die Wirtshäuser an den Straßen und in den Kleinstädten machten den deutschen, also auch preußischen Territorialherrschern gewisse Sorgen, was aus Edikten und Verordnungen hervorgeht ${ }^{3}$. Die preußischen Kurfürsten wiesen auf die Notwendigkeit hin, neue Gasthäuser mit Herbergsfunktion in den Kleinstädten und auf dem Lande gründen zu lassen. Die Kleinstadtbehörden sollten auch Mittel und Wege finden, Privathäuser in Gasthäuser zu verwandeln. Auf dem Lande sollte der künftige Investor mit steuerlichen Vergünstigungen dazu animiert werden. Zugleich stellten die Kurfürsten

1 Alle bibliographischen Angaben sind detailliert genannt in: W. Zientara, Sarmatia Europiana oder Sarmatia Asiana? Polen in den deutschsprachigen Druckwerken des 17. Jahrhunderts, (2003).

2 Des Nordischen Mercurij Verbesserter Wegweiser, von Zehen Haupt-Reisen aus der Stadt Hamburg, (1674); W. Zientara, Niemieckie media XVII wieku o Rzeczpospolitej szlacheckiej. Z szabla i kielichem, czyli życie po sarmacku, hg. v. J. Tazbir, S. Zagórski, (2015), S. 183-194.

3 Verordnung Des Hochgebornen Herrn, Herrn Heinrichen des Andern Jüngern und der Zeit Eltisten Reußen Herrn von Plauen, Herrn zu Graitz, Crannichfeld, Gerau, Schleitz und Lobenstein etc. [...], (1664), o.S.; Serenissimi Verordnung wegen der Gasthöfe in Dero Residenz=Stadt Wolfenbüttel, (1748), o.S.; W. Zientara, Essen unterwegs. Zwischen Angstgefühlen und Anerkennung für die Wirtshäuser auf Reisen in der Frühen Neuzeit, in: Documenta Pragensia, 25 (2007), S. 241-250. 
konkrete Forderungen in Bezug zum Beispiel auf die Umgebung, fließendes Wasser, Angebote für Menschen und Pferde. Dies präzisierte der Kurfürst von Brandenburg Friedrich III. am 14. Oktober 1697, also bevor er König in Preußen wurde. Er warb mit einer Steuerpolitik, bei der ein Unternehmer zunächst seine Herberge florieren lassen konnte und erst danach, nach der Einschätzung der durchschnittlichen Einkünfte, der vollen Steuerpflicht unterlag. Friedrich III. mahnte die Wirtsleute, Unhöflichkeit und grobes Benehmen zu vermeiden. Der Gast sollte wiederkommen und preußische Wirtshäuser in guter Erinnerung behalten. Der bisherige Zustand, „unsaubere und eckelhafte Häuser und Wohnungen" wurden nicht mehr geduldet. In den Edikten wiederholten sich die Appelle an anständiges Benehmen der Gäste und der Wirtsleute: „kein Schweren und Fluchen“, kein Glücksspiel, sondern bescheidenes Benehmen wurde erwartet, aber auch standesgemäße Kleidung der Frauen und Männer waren Äußerungsformen gottgefälligen Verhaltens, die in den Policeyordnungen regelmäßig wiederholt werden. Die Wirtshäuser oder ,anständigen' Dorfkrüge sollten voneinander zwei bis höchstens drei Meilen entfernt sein. Besonders schmerzhaft sollte der Wirt für jegliche Formen des Betrugs, aber auch für Ausschank von alkoholischen Getränken während des Gottesdienstes, an Fest- und Feiertagen bestraft werden. Der Gläubige hatte in dieser Zeit in der Kirche seinen Glauben zu demonstrieren und nicht im lokalen Dorfkrug zu trinken. Die Regeln waren klar und unmissverständlich, das Gesetz sah jedoch Ausnahmen für fremde Reisende und Kranke vor ${ }^{4}$.

Die Verordnung Friedrichs III. wurde mehrfach nachgedruckt. Ging es hier um Strafen, mit denen die Obrigkeit drohte, die kaum etwas bewirken konnten und ignoriert wurden? Wurden die lokalen Kontrollbeamten bestochen? Die rechtliche Lage der Gasthäuser, Dorfkrüge und Poststationen in den deutschen Territorialstaaten ist eine bunte Landschaft von Privilegien, lokaler Tradition, wiederholten Edikten, Kauf- und Verkaufsverträgen. Auch Universitäten profitierten davon. Die Universität Jena, die Salana, produzierte und verkaufte eigenes Bier und eigenen Wein, die im "Rosenhainischen Haus" und in der Umgebung der Stadt bereits im 16. Jahrhundert steuerfrei verkauft wurden. „Die Rose“ sicherte bedeutende

4 J. Rapoldt, Kirchen- policey- und process Ordnungen Deß Hochwürdigsten/ Durchläuchtigsten/ Hochgebornen Fürsten und Herrn Herrn/ Augusti, Postulirten Administratoris des Primats und Ertz-Stiffts Magdeburg/ Hertzogens zu Sachsen [...], (1652), S. 52. 
Einnahmen der Universität, sie war gut besucht, nicht nur während akademischer Feierlichkeiten ${ }^{5}$. Anfang des 18. Jahrhunderts brachte diese Schankwirtschaft der Universität 524 Gulden jährlich ein. „Die Rose“ war bei akademischen Konsumenten und den Jenaern überhaupt sehr beliebt, auch wenn viele Studenten eher die Wirtshäuser am Stadtrand oder gar weiter entfernte Wirtshäuser besuchten. Der Grund war eine lockerere Kontrolle seitens der Universität und die Gesellschaft von „Frauenzimmern “6. Trotz der Gefahr einer schmerzhaften Geld-Karzerstrafe oder des Verweises von der Universität riskierten sie diese Besuche, sonst hätten sie ihre Feier zu früher Stunde, um 21 bzw. um 22 Uhr, beenden müssen. Aber auch hier gab es eine Lücke in den rechtlichen Regelungen. Die mit Karzerhaft bestraften Studenten durften von ihren Kommilitonen reichlich mit Bier versorgt werden.

Auch Herzog Karl I. von Braunschweig-Wolfenbüttel äußerte seine Sorge (nicht nur) um die Studenten der Helmstädter Julius-Carls-Universität, die

[...] dadurch in mannigfaltige Händel und Unordnungen verwickelt worden, daß auf denen Kellern, Caffe-Schenken, und übrigen Wirths=Häusern bis in die späte Nacht, denen Verordnungen zuwider, Gesellschaften und Zechen von denen Wirthen geheget worden, dadurch aber nicht allein mancher derer ungezogenen Gäste seine Gesundheit mutwillig ruiniret, und sich zu der auf den andern Tag vorzunehmenden Arbeit untüchtig gemacht, sondern auch andere Mit=Einwohner in der Ruhe gestöhret, und solchen ein übles Exempel gegeben [...] daß in Zukunft auf denen Kellern, Caffee=Schenken, und andern Wirths=Häusern in und vor Unserer Stadt Helmstedt länger nicht, als des Abends bis 10, höchstens bis $11 \mathrm{Uhr}$ denen Studenten oder andern Gästen Bier, Wein, Brantwein oder Toback gereichet

5 S. Wallentin, Fürstliche Normen und akademische „Observanzen“. Die Verfassung der Universität Jena 1630-1730, (Veröffentlichungen der Historischen Kommission für Thüringen Kleine Reihe 27, 2009), S. 303-312.

6 J. Ch. Müller, Meines Lebens Vorfälle \& Neben-Umstände, 1: Kindheit und Studienjahre (1720-1746), (2007), S. 104, 110, 165-166; Zwischen Gotteshaus und Taverne. Öffentliche Räume in Spätmittelalter und Früher Neuzeit, hg. v. S. Rau, G. Schwerhoff, (2004); G. Schwerhoff, Die Policey im Wirtshaus. Obrigkeitliche und gesellschaftliche Normen im öffentlichen Raum der Frühen Neuzeit. Das Beispiel der Reichsstadt Köln, in: Machträume der frühneuzeitlichen Stadt, hg. v. Ch. Hochmuth, S. Rau, (1991) und die bisher achtbändige Serie der Quellenwerke: Die „gute“ Policey im Reichskreis, hg. v. W. Wüst. 
werde. Die Wirthe hergegen sollen schuldig und gehalten seyn, die Gäste schon um 10 Uhr, daß es Zeit sey heim zu gehen, zu erinnern, und nächstdem um halb 11 Uhr nochmals anzuzeigen, wie spät es an der Zeit sey. Gegeben auf unserer Vestung Wolfenbüttel den 20sten Martii $1747^{7}$.

\section{Soviel zur Wirksamkeit der Justiz!}

Karl I. stand unter dem Einfluss Friedrichs II., nahm am Siebenjährigen Krieg teil und danach geriet sein Herzogtum an die Grenze zum finanziellen Ruin. Die formulierte Sorge um die Gesundheit und das Wohlergehen seiner Untertanen ging, wie in Preußen unter Friedrich II., noch weiter. Der Herzog beschränkte den Handel mit Wein, Kaffee, Tee, Zucker auf diejenigen, die eine gültige Konzession hatten. Ausnahmen gab es jedoch u.a. für die Poststationen, damit der Reisende keine Unannehmlichkeiten (Diskomfort) erleiden mussten ${ }^{8}$. Wie alle Territorialherrscher vergaß Karl I. nicht, Denunzianten zu belohnen ${ }^{9}$. Karl I. engagierte sich für ein höheres Niveau der Dienstleistungen, denn davon profitierte jeder Staat, und suchte dies mit den Mitteln der Steuer- und „Behörden- und Policeykontrolle“ durchzusetzen. So ließ er im Sinne der Aufklärung die Wirte Zeitungen in den städtischen Gasthöfen auslegen ${ }^{10}$, um sie im gleichen Atemzug zu er-

7 Von Gottes Gnaden Wir Carl, Herzog zu Braunschweig und Lüneburg [...] Fügen hiemit zu wissen, (1747).

8 Braunschweigische Anzeigen, 20 (1764), S. 101-103; A. Hermeier, Postkutschen-Passagiere als privilegierte Kaffeetrinker, in: Archiv für Postgeschichte, 1 (1982), S. 94-95. Über die Kaffeeriecher Friedrich II. siehe: J. Schopenhauer, Ihr glücklichen Augen. Jugenderinnerungen. Tagebücher. Briefe, (' 1979$)$, insb. Kapitel: Im Würgegriff der Preußen, S. 87.

9 Braunschweigische Anzeigen, 20 (1764), S. 102: „Es soll aber davon bey 20.Thlr. Strafe von jedem Cantraventionsfall, welche halb den Denuncianten zu blligen, und halb der Obrigkeit anheim fällt, weder an die Dorfgemeinde gehörige Einwohner, noch an andere Landleute, es sey denn einheimische oder auswärtige, das mindeste verabfolget werden".

10 Vgl. auch: Policey- und cameral-magazin in welchem nach alphabetischer Ordnung [...], hg. v. J. H. L. Bergius, 4 (1769), S. 23-37, hier S. 26, Pkt. 9. Die Frage nach den Lesewelten, die vor und nach den Forschungsergebnissen von Chartier gestellt wurde (bzw. gestellt wird), ist immer noch aufregend und neue und alte Ansätze frischen Diskurse auf. Das Interesse für die Welt, für das gedruckte Wort, in Lesekreisen, wo jemand für die anderen laut gelesen hat, ist wohl intensiver gewesen als allgemein geglaubt wird. Vgl. R. Chartier, Lesewelten. Buch und Lektüre in der frühen Neuzeit, (1990), insb. Kapitel 4: Muße und Geselligkeit. Lautes Lesen in Europa der Neuzeit, S. 146-159. Die Zeitung. Deutsche Urteile und Dokumente von den Anfängen bis zur Gegenwart, ausgewählt und erläutert v. E. Blühm, R. Engelsing, (1985), S. 37: Krakeel im Wirtshaus 1656, unbekannter Korrespondent. 
mahnen, genauestens ihre Buchhaltung führen. Und auch die Kontrolleure hatte er im Blick:

Es ist nicht genug, daß die Policey gute Einrichtungen bey denen Gasthöfen veranstaltet, und deshalb Gesetze und Reglements publiciret; sondern sie muß auch über deren Beobachtung genaue Aufsicht haben. Diese Aufsicht ist das Notwendigste, ausser welcher die vortreflichsten Reglements und Taxen nicht die geringste Wirkung haben werden. Man verordnet zwar gemeiniglich, daß die Policeymeister die Gasthöfe öfters und unvermuthet visitieren sollen, um desto zuverlässiger zu wissen, wie es darinnen zugehe. Allein, thun dieses auch die Policeymeister allzeit so, wie sie sollten? Sie lassen es gemeiniglich darauf ankommen, bis sich die Reisenden selbst beschweren, und sie dadurch, so zu sagen, an die Visitation erst erinnern ${ }^{11}$.

Der Herzog dachte also an „die Taxe“, an die Kontrolle der guten Sitten, die Qualität der Dienstleistung. Zugleich wollte der Landesherr wissen, worüber im Gasthaus oder im dörflichen Krug gesprochen wurde, wer die Getränke verwässerte oder illegal verkaufte. Dieses Wissen betraf den Alltag des Wirtes, des Pächters, der lokalen Konsumenten und der fremden Reisenden, die sich immer wieder an neue Regeln anpassen mussten.

Interessant ist, dass die Kirche meistens nur in einem Kontext erwähnt wurde, Wirte durften während des Gottesdienstes und an Feiertagen keine alkoholischen Getränke verkaufen. Es sei denn ein Gast war krank oder ein Fremder auf der Durchreise, der nicht länger blieb und in Kürze seinen Geschäften nachgehen und das Wirtshaus verlassen würde ${ }^{12}$. Das Wirtshaus als öffentlicher Raum war für die lokale Obrigkeit einfach viel zu wichtig, um auf die Kontrolle der Untertanen zu verzichten. Die Wirte befanden sich selbst in einer schwierigen Position, wenn sie beispielsweise das Verhalten ihrer übernachtenden oder zechenden Gäste nicht beherrschen konnten. Schlägereien waren nicht selten, meist durch den Alkohol noch

11 Policey-und cameral-magazin, 4 (1769), S. 27, § 5.

12 A. Tlusty, Bacchus und die bürgerliche Ordnung. Die Kultur des Trinkens im frühneuzeitlichen Augsburg, 1; Studien zur Geschichte des Bayerischen Schwaben, hg. v. R. Kießling, 34 (2005), hier: S. 194-198: Das Wirtshaus als Ort der Kontrolle. 
angeheizt ${ }^{13}$. Diese Missstände waren bekannt, ihre Bekämpfung jedoch kaum wirksam, und die gedruckten Edikte der Territorialherren erinnerten eher daran als die unehrlichen Wirte und die Konsumenten tatsächlich abzuschrecken.

Nach dieser Einführung kommen wir jetzt zum Anfang des Aufsatzes zurück. Man kann annehmen, dass der reisende Jurist Johann Arnold Brand (1647-1691), ein Gelehrter vom Rang eines Johann Bernoulli, ein Geistlicher wie Johann Wendel Bardili, Johann Christian Fick, Daniel Chodowiecki und viele mehr, die wir nicht einmal vom Namen und aus gedruckten Berichten kennen, sich der Realität der deutschen Wirtshäuser und der rechtlichen Situation um diese öffentlichen Einrichtungen bewusst waren. Sie fuhren zunächst durch Pommern unter preußischer Justiz bis nach Stolp, und hier bekamen sie eine gutgemeinte Warnung: Wenn du in die Kaschubei kommst, bekommst du bis nach Danzig kaum etwas zu essen und kein Futter für deine Zugtiere ${ }^{14}$. Dieses Elend erlebte beispielsweise Chodowiecki, der voller Mitleid darüber schrieb und entsprechende Szenen zeichnete. Bardili, ein Geistlicher, reagierte hingegen mit Hochmut und Abscheu auf die Armut der Kaschuben. Wie viele andere waren Bardili und sein Prinz auf die Reise nicht richtig vorbereitet: Ihnen fehlte es an Sprachkenntnissen, lokalen Zahlungsmitteln und Lebensmitteln. Sie reagierten auf das Fremde mit Hohn und Spott. Auf dieser Strecke konnte man übrigens nur selten ein Wirtshaus finden. So atmeten die Reisenden auf, wenn sie endlich Danzig erreichten. Danzig, mit seinen hansischen Wurzeln, mit der vertrauten deutschen Sprache, garantierte ihnen eine problemlose Kommunikation und kulturelle Vielfalt in jeder Hinsicht. Kein Wunder, dass auch seine Wirtshäuser eine Erwähnung in den Reißbüchlein des 17.

13 K. Simon-Muscheid, Die Dinge im Schnittpunkt sozialer Beziehungsnetze: Reden und Objekte im Alltag (Oberrhein, 14. bis 16.Jahrhundert), (2004), S. 267-282, besonders das Kapitel: Was jenseits der Wand geschieht, geht dich nichts an. Formen wirtshausspezifischer Delinquenz.

14 J. W. Bardili, Des Weyland Durchl. Printzens Maximilian Emanuels Hertzogs in Würtemberg etc. Obristen über ein Schwedisch Dragoner Regiment Reisen und Campagnen durch Deutschland in Polen, Lithauen, roth und weiß Reußland, Wolhynien, Severien und Ukraine [...], (1730); J. P. Graffenauer, Meine Berufsreise durch Deutschland, Preußen und das Herzogthum Warschau, in den Jahren 1805, 1806, 1807 und 1808, (1811). Jean-Philippe Graffenauer (1775-1838) bestätigt diese Information, mit dem Unterschied, dass man sich mit Lebensmitteln und Futter für die Pferde ein Stück weiter, in Bütow versorgen sollte. Hier: S. 130. 
und 18. Jahrhunderts fanden. Hätte Bardili zum Beispiel das Büchlein von Lehmann gelesen, hätte er sich und seinen mitreisenden Kommilitonen viel Kummer ersparen können ${ }^{15}$.

Auch in Danzig wollten die städtischen Behörden schnell und detailliert von den Wirten erfahren, wer soeben angekommen war, was der Zweck seines Aufenthaltes sei und wie lange er in der Stadt bleiben wollte. Der erwähnte J. Bernoulli beispielsweise besuchte Bibliotheken, private Buchsammlungen, unterhielt sich mit seinesgleichen und kümmerte sich kaum um die Wirtshäuser. Eine der wenigen Ausnahmen in seinen Reiseaufzeichnungen galt dem gewählten Danziger Wirtshaus, dem englischen Hause ${ }^{16}$. Ebenso wie Bernoulli war auch Johann Christian Fick wenig begeistert von den Danziger Wirthäusern und das ist ein bedeutender Unterschied zum 17. Jahrhundert. Die Meinung von Fick war deshalb so folgenreich, weil er Verfasser von Reiseführern war ${ }^{17}$. Ein anderer Reisender, Giacomo

15 [P. A. Lehmann], Die vornehmsten Europaeischen Reisen/ Wie solche durch Deutschland, Frankreich, Italien, Holl- und Engelland, Dänemark und Schweden, Vermittelst der darzu verfertigten Reise-Carten nach den bequemsten Post-Wegen anzustellen [...], (1713), S. 133: „Dantzig: Logiret in der Königsberger Herberge, in der Kayserl. Herberge, in güldenen Huffeisen, drey Mohren, in dem München aufm Holtz-Markt, Schipper-Gilde Hauß, auf der H.Geist Gasse“, S. 136: „Königsberg: Wirths-Häuser finden sich überflüssig, insonderheit sind zu recommendiren der Polnische Krug, in der alten Stadt, in Lobenicht der Palmbaum, in Kniephoff sind das weisse Roß, der Bären und Löwen-Krug die besten“.

16 J. Bernoulli, Johann Bernoulli's Reisen durch Brandenburg, Pommern, Preußen, Curland, Rußland und Polen in den Jahren 1777 und 1778, 1 (1797), zweite Abtheilung, Aufenthalt in Danzig , S. 151: „Ich stieg in Danzig bey dem englischen Hause ab, ein Wirthshaus, das mir als das beste war gerühmt worden, von welchem ich aber wenig gutes sagen kann. Mit der Bewirthung war ich ziemlich zufrieden, etwas mehr Reinlichkeit aber hätte ich gewünscht“.

17 J. Ch. Fick, Meine neueste Reise zu Wasser und zu Land oder ein Bruchstück aus der Geschichte meines Lebens, (1807), S. 133-135: „Man staunt, daß in einem so hoch kultivierten Staate, wie der preussische ist, auf einer der größten Strassen, von der Haupt= und Residenzstadt der ganzen Monarchie Berlin, zur Hauptstadt des Königsreichs, Königsberg, und zu der wichtigsten Handelsstadt, Danzig, für die große Menge von Reisenden so gar wenig gesorgt ist. Die an der Strasse und in den seltenen Dörfern hie und da befindlichen Wirthshäuser, oder sogenannten Krüge, sind ein Inbegriff von Schmutz und schlechter Wirtschaft. Hier ist äussert selten für die Bequemlichkeit und für ein nur etwas erträgliches Nachtlager gesorgt; Essen und Trinken, wenn man noch welches erhalten kann, sind von der schlechtesten Gattung; selbst der Kaffe wird gewöhnlich so schlecht zubereitet, daß man ihn kaum genießen kann“. Und weiter: „Dieses Stück Land, ich meine die Herrschaften Lauenburg und Bütow, oder einen Theil des ehemaligen Pomerellens, trägt noch ganz den Karakter von Polen, zu dem sie ehemals eine Zeitlang gehörten, an 
Fantuzzi, kam aus Warschau und fuhr durch Pommern um den ,protestantischen Teufel' im Menschen kennen zu lernen. Als Katholik im Dienste des Nuntius reiste er durch die protestantischen Länder nach Rom, hatte Angst sich am Tisch im Wirtshaus zu bekreuzigen und machte sich Gedanken, ob ein heimtückischer Wirt ihm am Freitag nicht etwa eine Schweinebrühe auftischte. Eine wahre Attraktion, die nicht jedem Fremden angeboten wurde, war die Besichtigung der Festung Weichselmünde ${ }^{18}$.

Sehen wir uns die Regelungen des Danziger Wettgerichts oder die Edikte des Stadtrates an, so zeigt sich, dass mehrere Elemente wie auch die antizipierten Vergehen der Wirte und der Gäste ähnlich denen in den deutschen Territorialstaaten waren ${ }^{19}$.

Wein-Meth-Caffee-Bier und Brantwein-Häuser, Keller, Krüge, oder Buden, wie auch Garküchen an Sonn- und andern hohen Feyertagen unter dem Gottesdienst, nemlich des Morgens von 8 bis 10, und von 12 bis 1, imgleichen von 2 bis 4 Uhr Nachmittage, eröfnen, bey 6 Fl. Strafe. Auch soll an Sonn- und hohen Fest-Tagen kein Wirth seine Gäste des Abends länger sitzen lassen, und ihnen Wein, Meth, Bier, Thee, Caffee, Brantwein und anderes Getränke geben, als im Sommer, nemlich von Ostern bis Michaelis, bis $11 \mathrm{Uhr}$, und im Winter, nemlich von Michaelis bis Ostern, bis $10 \mathrm{Uhr}$ bey Strafe $18 \mathrm{Fl}$., oder dreytägiger Gefängniß [...] (S.170).

sich. Nur fehlt in einer Ecke der Stube die Tonne mit Sauerkraut, aus welcher jedes Glied einer Polnischen Familie gleich beim Aufstehen, mit ungesäuberten Händen durch einige Griffe in dieselbe, sein Frühstück heraus holt, wodurch der Gährungsstoff wegen der neuen frembdartigen Ingredienzien vermehret wird“. Dieses Zitat gibt es auch in meinem Aufsatz: W. Zientara, Reise nach Danzig Anfang des 19. Jahrhunderts, in: Hybride Identitäten in den preußisch-polnischen Stadtkulturen der Aufklärung. Studien zur Aufklärungsdiffusion zwischen Stadt und Land, zur Identitätsbildung und zum Kulturaustausch in regionalen und internationalen Kommunikationsnetzwerken, hg. v. J. Kodzik, W.Zientara, (2016), S. 261-274.

18 E. Kizik, Die Gäste der Festung Weichselmünde bei Danzig im 17. und 18. Jahrhundert, in: Reisen, Wahrnehmen, Kommunizieren im deutsch-polnischen Kontext in der Frühen Neuzeit. Festschrift zum 65.Geburtstag von Prof. Włodzimierz Zientara, hg. v. J. Kodzik, A. Mikołajewska, (2019), S.71-78; J. Kodzik, Im Wirtshaus und in der Postkutsche. Herrnhutische Kommunikationsstrategien auf Reisen am Beispiel Polen-Litauens, in: Reisen, Wahrnehmen, Kommunizieren, S. 79-98.

19 Neu-revidirte Willkühr der Stadt Danzig, aus Schluß Sämmtlicher Ordnungen, (1761), S. 66, 110-111, 143, 170-171; E. Kotarski, Regulacje prawne osiemnastowiecznego Gdańska dotyczace gospód i kawiarni, in: Rzeczpospolita domów, 5: Karczmy zajazdy, gospody, hg. v. K. Krawiec-Złotkowska, (2019), S. 15-24. 
Der Danziger Stadtrat hatte mehr Verständnis für die Bedürfnisse von Gästen und Wirten an Festtagen und erlaubte unter konkreten zeitlichen Beschränkungen den Genuss von Getränken im Wirtshaus. Jedoch waren alle Formen von Theaterspielen, Tanzen, Spielen unter Strafandrohung von $30 \mathrm{Fl}$. verboten. Desgleichen wurden Glückspiele jeglicher Art, Wein- und Bierverfälschung durch die Wirte sowie Alkoholschmuggel durch die Schiffsbesatzungen strengstens in den wiederholten Edikten verboten. Besonders heftig reagierte der Stadtrat in allen Fällen, in denen er als städtischer Zensor auftrat. Gedruckte Schmähschriften wurden nicht toleriert, deshalb riskierten manche Autoren, sie als Handschriften zu verbreiten. Zeitungen unterlagen auch der städtischen Kontrolle, man kann also vermuten, dass das Membersche Kaffeehaus, wo sie ausgelegt waren, von den Argusaugen des Zensors beobachtet wurde ${ }^{20}$. So wie in Polens Nachbarland Preußen, im Norden und Süden Deutschlands war wohl die staatliche Kontrolle nicht so wirksam, wie es die Obrigkeit wünschte. Aber diese Kontrolle wurde ausgeübt, die Beamten kannten ihre Pflichten, denen sie mit unterschiedlichem Eifer nachgegangen sind. Das geschriebene, gedruckte, vervielfältigte Recht wurde durch mahnende Bekanntmachungen ausgehängt. Der fremde Reisende, der sich in Danzig entschied, längs der Weichsel zu fahren, verließ die Grenzen Königlich Preußens und lernte eine andere Wirklichkeit kennen. „Die Wirtshäuser in Polen hielten sich an eige-

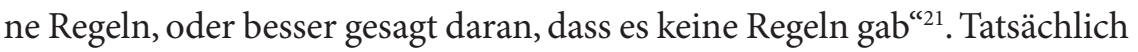
boten das Wirtshaus bzw. ein Dorfkrug nicht nur keinen Komfort, im 17. Jahrhundert eventuell nur ein Dach über dem Kopf und nichts weiter. Der Gast hatte Glück, wenn sein Pferd in Sicherheit war und gefüttert wurde. Hatte der Gast keine eigenen Vorräte, musste er hungern, und der Wirt oder der jüdische Pächter bot eventuell nur Alkohol von schlechter Qualität. Das Wirtshaus unterstand meistens dem lokalen adeligen Hof oder dem dörflichen Probst. Die überall herrschende Armut war verursacht durch eine Reihe von Kriegen, schlecht oder gar nicht besoldete ehemalige Soldaten zogen durch das Land und raubten auch die wehrlosen Wirte aus. Der polnische Adelige als Gast hielt es für unter seiner Würde, die Rechnung

20 Kotarski, Regulacje prawne, S. 18; Kawiarnia Mombera, in: Gedanopedia, URL: https://www.gedanopedia.pl/gdansk/?title=KAWIARNIA_MOMBERA (31.05.2020).

21 M. Mielnik, Siedlisko grzechu, czyli karczmy w sztuce gdańskiej na tle sztuki europejskiej w XVI i na początku XVII wieku, in: Rzeczpospolita domów, (2019), wie Anm. 19, S. 26-34. 
für das Futter und die Lebensmittel zu bezahlen. Was florierte, war die Bierund Branntweinherstellung, entweder an Ort und Stelle oder bezogen von der benachbarten Stadt, wenn der Wirt es durfte. Das Wirtshaus war im 17. Jahrhundert noch ein hölzernes Gebäude, es bestand aus der großen Stube und ein paar benachbarten kleinen Kammern sowie einer Feuerstelle zum Kochen. In den Berichten besonders des 18. und vom Anfang des 19. Jahrhunderts wurde oftmals mit Spott ein Fass mit Sauerkraut am Eingang zum Wirtshaus erwähnt, von dem ein penetranter Gestank ausging, der nicht auszublenden war. Dieses Bild verbreiteten beispielsweise Ulrich von Werdum, Paul J. Marperger, der schon erwähnte Fick und viele anonyme Berichterstatter ${ }^{22}$. Sie betonten, unabhängig von der Epoche, welch' emsige Wirte, Boten und Dolmetscher insbesondere Juden waren. Wenn sie ihnen schmutzige Wirtshäuser, in denen sie auf dem nackten Boden schlafen mussten, vorwarfen und ihre Ekelgefühle demonstrierten (siehe dazu auch die französischen Berichte über das Judenviertel von Krakau ${ }^{23}$ ), schüttelten sie mit Verwunderung den Kopf. Auch das Engagement des Klerus in den Wirtshäusern und Dorfkrügen wie in der Alkoholproduktion war für sie etwas Seltsames. Das findet sich in den deutschen Quellen, die im ersten Teil des Aufsatzes genannt wurden, nicht. Es wurde, wie in Danzig auch, der Gottesdienst erwähnt und das Verbot, währenddessen Alkohol zu verkaufen. Inhaber von Wirtshaus oder Dorfkrug konnten Adelige, ein Probst oder der Schulze bzw. Schultheiß sein. Interessant erschien in Polen das intensive Engagement der Geistlichen, sowohl als Alkoholproduzenten wie auch als Konsumenten, ihre Teilnahme an Trinkgelagen mit den Bauern, ungeachtet ihrer Position und Rolle in der Gesellschaft ${ }^{24}$.

22 U. von Werdum, Das Reisejournal des Ulrich von Werdum (1670-1677), in: Kritische Edition eines Reiseberichtes, hg. v. S. Cramer, (1990); P. J. Marperger, Königlich-Polnisch. und Chur-Sächsisch. Hof- und Commercien-Rahts, wie auch Mitglieds der Königlich-Preußischen Societät der Wissenschafften, Vollständiges Küch- und Keller-Dictionarium, (1717), S. 926; Handbuch für Reisende in Dänemark, Norwegen, Schweden, Russland, Polen und Finnland von DR. Karl Theodor Wagner [Hg.] . Eine Fortsetzung zu C.G.D. Stein's Reisen nach den Hauptstädten von Mittel=Europa, (1840), S.143; Auszüge aus dem Tagebuch von einer Geschäftsreise nach Rußland im Jahr 1805 mit beigefügten ganz genauen Postrouten, (1812), S. 104-107; W. Coxe, Reise durch Polen, Rußland, Schweden, Dänemark. Mit historischen Nachrichten, und politischen Bemerkungen begleitet, 1 (1785), S. 165: ebenda, 2 (1786).

23 E. M. Szarota, Die gelehrte Welt des 17. Jahrhunderts über Polen, (1972).

24 J. Kotarska, „Karczem dwie krzyżowego gościńca pilnują". Społeczne przestrzenie staropolskiej karczmy, in: Rzeczpospolita domów, (2019), S. 89. 
Mit der Zeit wurde der Besitz in Pacht verwandelt. Juden galten als bequeme Pächter, die Alkohol verkauften, für die Ordnung unter den Gästen sorgen sollten und den Bauern Kredite gewährten, denn für den katholi-

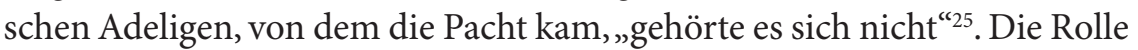
eines polnischen Dorfkruges ist auch dadurch nicht zu überschätzen, da ein Bauer nur mit Schwierigkeiten sein Dorf verlassen durfte, es sei denn, es war Jahrmarkt in der benachbarten Stadt ${ }^{26}$. Auch sein Geld gab er nur im lokalen Krug aus, denn das war der Sinn des propinatio: Der Inhaber oder Pächter einer Dorfbrennerei hatte einen dauerhaften finanziellen Gewinn durch den Verkauf in der Dorfschenke. Die Untertanen, mit wenigen Ausnahmen, durften ihr Dorf nicht verlassen. Ihre Welt war dadurch sehr eingeschränkt. Umso mehr war jeder fremde Gast in der Schenke gern gesehen. Er erzählte von der nahen und fernen Welt, die die Bauern nicht kannten. Am Ende war der jüdische Pächter am eventuellen Unglück eines Bauern schuld und nicht der Adelige. So zahlten dafür viele Juden während der Pogrome um die Mitte des 17. Jahrhunderts in der Ukraine mit ihrem Leben.

Das Angebot der meisten Wirtshäuser war mehr als schlecht. Ein Reisender zog oft die Übernachtungsmöglichkeit im Stall mit seinen Pferden, auf Heu oder Stroh der Stube nebenan, mit Gestank und Ungeziefer als Schlafgefährten, vor. Ein Bett war in einem Dorfwirtshaus weitgehend unbekannt. Deshalb warnte man die fremden Reisenden: Wenn du nach Polen gehst, musst du ein Bett dabei haben. Adelige waren, wenn sie Fremde an ihrem Hof aufnahmen, großzügige Gastgeber, aber sie hatten nur ein Bett für sich und ihre Gemahlin. Derartige Warnungen waren zwar eine Mischung von Dichtung und Wahrheit, aber das Stereotyp funktionierte und hielt sich hartnäckig ${ }^{27}$. Das negative Bild der Wirtshäuser wurde auf die gesamte Nation übertragen. In seinen Vorlesungen für die Göttinger

25 Ebenda, S. 82-97.

26 Die neueste Forschung über die Migration vom Lande in die Stadt wird wohl diese Feststellung allmählich ändern, vgl. M. Wyżga, Ze wspótczesnych badań nad mobilnościa w Europie przednowoczesnej, in: Przeszłość Demograficzna, 40 (2018), S.11-37, ders., Homo movens. Mobilność chłopów w mikroregionie krakowskim w XVI-XVIII wieku, (2019); A. Walaszek, Migracje Europejczyków 1650-1914, (2007).

27 G. B. Fagiuoli, Diariusz podróży do Polski (1690-1691), (2017), S. 76-78: „Auf der Reise aus Wien Richtung Norden finde man keine Betten. Eine Matratze, Bettwäsche sollte man unbedingt dabei haben". 
Studenten wiederholte der Hofrat August Ludwig Schlözer die Relevanz der Kenntnisse von Fremdsprachen ${ }^{28}$, denn Unkenntnis der Sprache und des fremden $\mathrm{zu}$ besuchenden Landes verursache, dass man auf Reisen letztendlich verzichte. Stellen wir jetzt die Frage, wie viele deutsche Reisende wir kennen, die verkündet haben, dass sie Polnisch lernten, weil sie das Land und die Menschen besser kennen lernen und sich aus eigener Anschauung eine Meinung bilden wollten, Zeitungen auf Polnisch lesen, mit ihresgleichen sich auf der Straße und im Wirtshaus auf Polnisch unterhalten wollten. Wir sprechen hier nicht von Vermutungen, weil es seit Gutenberg gleich die ersten Sprachbücher gab, sondern von bewussten schriftlichen Quellen konkreter Menschen. Dem Verfasser ist ein einziges Beispiel bekannt.

Bis zu den Teilungen von Polen haben wir es mit einer Situation zu tun, für die man unterschiedlichste Beispiele nennen kann. Nachdem aber preußische Beamte in das sog. Südpreußen kamen, als sie Thorn, Danzig, Plock, Warschau besetzt hatten und bildlich gesehen die Fahne der, wahren Kultur' und ,Zivilisation' schwangen, begann im Schrifttum eine neue Epoche. Man musste vor sich selbst und der Welt rechtfertigen, dass es legitim sei, Polen von der europäischen Landkarte zu radieren ${ }^{29}$. So findet sich bei Krünitz zum Stichwort „Trinken, Trinksucht“ ein Aufsatz von 160 Seiten! ${ }^{30}$ Zum Stichwort Polen wurde als Experte Carl Theodor Uklanski ausgiebig zitiert $^{31}$. Krünitz stellte jedoch den abscheulichen Berichten Uklanskis über

28 A. L. Schlözer, Entwurf zu einem Reise=Collegio, (1777); F. Stoud, Das Reise-Collegium des Hofrat Schlözers gehalten im Winter 1792-1793, in: U. Kutter, Reisen - Reisehandbücher - Wissenschaft. Materialien zur Reisekultur im 18. Jahrhundert, (1996), S. 335-371, hier: 343 und 357: „Wo Menschencultur aufhört, hören Posten \& Zeitungen auf“, S. 367: „Ohne Gasthöfen kann man sich schwerlich eine Welt gedenken, wo gereist ist. Sie sind ein wahres Zeichen der Cultur des Landes“.

29 K. Holste, Das Wechselspiel von Fremd und Selbstexotisierung polnisch-litauischer Eliten im letzten Drittel des 18. Jahrhunderts, in: Konstruktionen Europas in der Frühen Neuzeit. Geographische und historische Imaginationen, hg. v. S. Richter, M. Roth, S. Meurer, (2017), S. 97-115, hier: S. 102-105.

30 J. G. Krünitz, Johann Georg Krünitz' ökonomisch-technologische Encyklopädie oder allgemeines System der Staats-, Stadt-, Haus-, und Landwirtschaft und der Kunstgeschichte, 188 (1846), S. 100-260, über Polen: S. 208-213.

31 C. T. Uklanski, Briefe über Polen, Österreich, Sachsen, Bayern, Italien, Etrurien, den Kirchenstaat und Neapel, an die Comtesse Constance de S., 1 (1808); vgl. seine Biografie: R. Straubel, Biographisches Handbuch der preußischen Verwaltungs- und Justizbeamten 1740-1806, Biographien, 2: M-Z, (2009), S. 1034. 
Polen das Buch von Bornschein gegenüber ${ }^{32}$. Bornschein war zwar kritisch, aber seine Aussagen waren nicht im gleichen Maße mit Anfeindungen gespickt:

Der Pole liebt den Trunk mehr als alles. Er übertrifft hierin fast noch unsere Vorfahren, die alten Teutschen. Ueber ein Trinkgelage geht ihm kein Genuß. Auch die Geistlichkeit macht hierin keine Ausnahme und die Advokaten fangen ihre Prozesse mit der Weinflasche an und endigen sie damit. Die schlechte Beschaffenheit der öffentlichen Gasthäuser ist oft die Ursache, daß die Reisenden ein Nachtquartier in irgend einem adlichen oder andern Hause suchen. Sie kennen den Besitzer nicht, sie sehen ihn zum erstenmahl, und mit Zuvorkommenheit und Liebe nimmt sie derselbe auf ${ }^{33}$.

Preußische Beamte wie Uklanski, Ernst Theodor Amadeus Hoffmann, Johann Friedrich Theodor Baumann oder Johann Friedrich Benedict Schartow, fuhren in dieses fremde Land, meistens direkt nach dem Jurastudium. Mehr oder weniger litten sie - wie Hoffmann in der, Verbannung in Płock, dem es noch dazu ständig an Geldmitteln fehlte. Er fühlte sich gedemütigt und träumte von einer Italienreise. Schartow war unter diesen Beamten eine Ausnahme. Er war es auch, der das Erlernen der schwierigen polnischen Sprache als harte Arbeit in seinem Büchlein beschrieb ${ }^{34}$. Aber welchen Zusammenhang gibt es hier mit einem Wirtshaus? Schartow fuhr mit seiner soeben angetrauten Ehefrau aus Berlin nach Warschau, um dort längere Zeit zu arbeiten. Die junge Dame, auch unter dem Einfluss eines ebenso jammernden Kutschers, geriet immer mehr in Panik, da sie die Sicherheit des ihr vertrauten Idioms verlor. Bis Posen hörte sie noch das ihr vertraute Deutsch. Auf dem weiteren Wege aber hörte sie schon eine fremde Sprache, der Kutscher jammerte laut vor Angst und sie verlor letztendlich die Beherrschung. Die Reisenden kamen in ein Wirtshaus,

32 E. Bornschein, Geschichte von Polen vom Ursprung dieses Reichs an bis auf die neuesten Zeiten, (1808), S.4-31.

33 Ebenda, S. 4.

34 [J. F. B. Schartow], Die Polen. Ein Beitrag zur Charakteristik dieses Volkes aus den Erinnerungen eines alten Justiz-Beamten, (1848). Auszüge aus diesem Buch erschienen in meinem Aufsatz: W. Zientara, Die Wahrnehmung Polens in den deutschen Kalendern des 17. Jahrhunderts, in: Schreibkalender und ihre Autoren in Mittel-, Ost- und Ostmitteleuropa (1540-1850), hg. v. K.-D. Herbst, W. Greiling, (2018), S. 267-279, hier: S. 268-269. 
bereits am Abend, in dem die Bauern tanzten und sangen. Die Dame wollte jedoch lieber über Nacht weiter fahren als dort zu übernachten. Denken wir in dieser Situation an die Vorlesungen von Schlözer! Schartow arbeitete in Warschau hart, war nach seiner eigenen Meinung ein vorbildlicher Beamter, der wie seine Kollegen durch das eingeführte preußische Recht ,wahre Zivilisation' in das besetzte Polen brachte. Für diese Mission opferte er sogar sein Familienleben.

Die Antwort auf die Frage nach der Selbstreflexion der Polen kann nur unbefriedigend sein. Nur ungern wird man sich überlegen wollen, inwieweit die polnischen Eliten an den Teilungen Polens beteiligt waren. An Kritikern fehlte es nicht. Wer aber wollte auf Sebastian Fabian Klonowic, Krzysztof Opaliński oder Wacław Potocki hören ${ }^{35}$ ? Die Schwächen der Polen wurden von den drei Nachbarstaaten ausgenutzt, aber jede Diskussion darüber sollte man eben mit diesen Schwächen anfangen, bevor man sich über die Besatzer empört. Sie arbeiteten emsig daran, dieses Polen, das es nicht mehr als Staat gab, in Dutzenden und Aberdutzenden Druckwerken darzustellen, auch als eine Nation, der man vermitteln müsse, was wahre Kultur sei ${ }^{36}$.

Ich ging in die Küche, um nach der Arbeit unseres Koches zu sehen. Hier hatte ich Gelegenheit, einen echt polnischen Koch in seinen Beschäftigungen zu beobachten. Diesen gesehen zu haben, und doch von seinen Speisen essen zu können, dazu hätte der wüthendste Hunger, und doch noch Überwindung gehört. Der Koch, oder wie ihn die Polen nennen, Kukars, war ein kurzer, stämmichter Mensch, der auf seinen bloßen Leib kein Hemd, sondern braunen Tuche gezogen hatte. Sein Gesicht und seine Hände waren der Farbe nach von dem Tuche nicht zu unterscheiden, und das ganze Männchen war mit Ruße getucht. Dabei trug er einen Schnurrbart, die übrigen Barthare waren aber seit vierzehn Tagen gewiss nicht abgeschoren worden, wodurch das schmutzige Gesicht, an dem große Schweißtropfen hingen, noch ekelhafter wurde. Er hatte ein Stück Sackleinwand als Schürze vorgebunden. Seine Füße waren nicht mit Schuhen

35 J. Kotarska, „Karczem dwie krzyżowego gościnca pilnuja“; H. Orłowski, „Polnische Wirtschaft“. Zum deutschen Polendiskurs der Neuzeit, (1996), S. 386-403.

${ }^{36}$ Meine Reisen durch einen Theil der preußischen Staaten, damaliges Gallizien, Schlesien, Mähren, Böhmen, Sachsen und Mecklenburg, 2 (1818), S. 187-188. 
und Strümpfen bekleidet; aber man war doch zweifelhaft, ob er in nackten Füßen dort stände.

Was haben solche bildhaften Darstellungen bewirkt? Die Diskriminierung begann bereits in der Schule: „Der Polak ist ein starker und herzhafter Mann. Er trinkt gern Brantwein und andere starke Getränke. Er trägt gern einen Knebelbart, und einen Säbel an der Seite“37.

Sicherlich galten für die Gasthöfe in den größeren Städten andere Bedingungen als für die Krüge und Wirtshäuser auf dem Lande. In der Stadt gab es eine strengere Kontrolle der Behörden, die Wirtshäuser waren von Gästen besucht, die ungern auf dem Lande übernachten wollten. Wenn die Routenabschnitte gut geplant waren, hatte ein wohlhabender Reisender weniger Kontakt zu dieser stereotyp negativ dargestellten Außenwelt der Bauern, polnischen und jüdischen Pächter, zu rohen Sitten und brutalen Verhaltensweisen, die durch Alkoholkonsum verstärkt wurden. Diese Kontrolle war in den deutschen Ländern intensiver, obwohl nicht so wirksam, wie die lokalen Obrigkeiten wünschten. Es stellt sich auch die Frage nach Vorbildern auf dem Lande und in der Stadt. Ist ein betrunkener Geistlicher, der mit den Bauern in einem polnischen Dorfkrug zecht, ein Vorbild? Wie steht dazu überhaupt die Tatsache, dass er der Inhaber oder Pächter des Wirtshauses ist? Am nächsten Tag könnte er vielleicht noch angetrunken vor dem Altar stehen, der nur wenige Meter vom Dorfkrug entfernt ist. Die dem deutschen Reisenden bekannten Verordnungen, Mahnungen, also Kenntnisse der eigenen Realität im Reich, Kenntnisse der Kriminaltaten und Vergehen in und um die Wirtshäuser führen zu keiner kritischen Eigenreflexion, als sie über das Nachbarland schrieben. Der bequeme, ständig wiederholte Mechanismus funktionierte nach wie vor: Uns geht es besser, dem Nachbarn fatal. Und so soll es auch bleiben, glaubte man ${ }^{38}$.

37 G. Ch. Raff, Geographie für Kinder, $1\left({ }^{4} 1787\right)$, S.405.

38 H. H. Hahn, E. Hahn, Nationale Stereotype. Plädoyer für eine historische Stereotypenfoschung, in: Stereotyp, Identität und Geschichte, hg. v. H. H. Hahn, (2002), S. 17-56. 


\section{ZARZĄDZENIA I STEREOTYPY}

NIEMIECKIE I POLSKIE GOSPODY W OKRESIE WCZESNONOWOŻYTNYM

STRESZCZENIE

Karczmy, jako ważne centrum komunikacyjne, podlegały specjalnej kontroli. W artykule omówiono dotyczące ich zarządzenia i edykty niemieckich władców terytorialnych. Nieco inaczej kształtowała się sytuacja gościńców w Polsce. Podróżnicy pruscy (oraz z dalszych terenów Rzeszy) przybywali do tego kraju przekonani o własnej wyższości kulturowej. Oferta polskich gospód i wiejskich karczm była wówczas rzeczywiście marna, ale krytykę rozciągano na kulturę całego kraju. Po trzech rozbiorach Polski stereotypy były chętnie rozpowszechniane i utrwalane przez pruskich urzędników.

\section{VERORDNUNGEN UND STEREOTYPE \\ DEUTSCHE UND POLNISCHE WIRTSHÄUSER IN DER FRÜHEN NEUZEIT}

ZUSAMMENFASSUNG

Als wichtiges Kommunikationszentrum unterlagen die Wirtshäuser besonderer Kontrolle. Im Aufsatz werden die sie betreffenden Verordnungen und Edikte der deutschen Territorialherrscher besprochen. Die Situation der Gaststätten in Polen war etwas anders. Die preußischen Reisenden (und diejenigen aus dem weiteren Deutschen Reich) kamen in das Land und waren von der eigenen kulturellen Überlegenheit überzeugt. Das Angebot der polnischen Wirtshäuser und Dorfkrüge war damals tatsächlich miserabel, die Kritik wurde aber auf die gesamte Kultur des Volkes ausgedehnt. Nach den drei Teilungen Polens wurden die Stereotype durch preußische Beamte gerne vermehrt und gefestigt.

\section{ORDERS AND STEREOTYPES}

GERMAN AND POLISH TAVERNS IN THE EARLY MODERN PERIOD

SUMMARY

Taverns, as an important communication center, were subject to special control. The article discusses the ordinances and edicts of German territorial rulers. The situation of highways in Poland was slightly different. Prussian travellers (and travellers from further areas of the Reich) came to this country convinced of their own cultural superiority. At that time, the offer of Polish taverns and country inns was really poor, but the criticism was extended to the culture of the entire country. After the three partitions of Poland, such stereotypes were readily disseminated and perpetuated by Prussian officials.

Translated by Agnieszka Chabros 


\section{SŁOWA KLUCZOWE / SCHLAGWORTE / KEYWORDS}

- gospoda; stosunki polsko-niemieckie; stereotyp; uprzedzenia

- das Wirtshaus; deutsch-polnische Beziehungen; Stereotype; Vorurteile

- tavern; Polish-German relations; stereotype; prejudice

\section{BIBLIOGRAFIA / BIBLIOGRAFIE / BIBLIOGRAPHY}

\section{ŹRÓDŁA DRUKOWANE / GEDRUCKTE QUELLEN / PRINTED SOURCES}

Auszüge aus dem Tagebuch von einer Geschäftsreise nach Rußland im Jahr 1805 mit beigefügten ganz genauen Postrouten, (1812).

Bardili J. W., Des Weyland Durchl. Printzens Maximilian Emanuels Hertzogs in Würtemberg etc. Obristen über ein Schwedisch Dragoner Regiment Reisen und Campagnen durch Deutschland in Polen, Lithauen, roth und weiß Reußland, Wolhynien, Severien und Ukraine [...], (1730).

Bergius H. L. Policey- und cameral-magazin in welchem nach alphabetischer Ordnung [...], 4 (1769).

Bernoulli J., Johann Bernoulli's Reisen durch Brandenburg, Pommern, Preußen, Curland, Rußland und Polen in den Jahren 1777 und 1778, 1 (1797).

Braunschweigische Anzeigen, 20 (1764).

Coxe W., Reise durch Polen, Rußland, Schweden, Dänemark. Mit historischen Nachrichten, und politischen Bemerkungen begleitet, 1-2 (1785, 1786).

Des Nordischen Mercurij Verbesserter Wegweiser, von Zehen Haupt - Reisen aus der Stadt Hamburg, (1674).

Die "gute" Policey im Reichskreis. Zur frühmodernen Normensetzung in den Kernregionen des Alten Reiches. Ein Quellenwerk, hg. v. W. Wüst, 1-8 (2001-2018).

Die Zeitung. Deutsche Urteile und Dokumente von den Anfängen bis zur Gegenwart, (1985).

Entwurf zu einem Reise=Collegio von A. L. Schlözer Prof. In Göttingen, nebst einer Anzeige seines Zeitungs=Collegii, (1777).

Fagiuoli G. B., Diariusz podróży do Polski (1690-1691), (2017).

Fick J. Ch., Meine neueste Reise zu Wasser und zu Land oder ein Bruchstück aus der Geschichte meines Lebens, (1807).

Graffenauer J. P., Meine Berufsreise durch Deutschland, Preußen und das Herzogthum Warschau, in den Jahren 1805, 1806, 1807 und 1808, (1811).

Hahn K., Meine Reisen durch einen Theil der preußischen Staaten, damaliges Gallizien, Schlesien, Mähren, Böhmen, Sachsen und Mecklenburg [...], 2 (1818)

Handbuch für Reisende in Dänemark, Norwegen, Schweden, Russland, Polen und Finnland [...], (1840).

Kirchen- policey- und process Ordnungen Deß Hochwürdigsten/ Durchläuchtigsten/ Hochgebornen Fürsten und Herrn Herrn/ Augusti, Postulirten Administratoris des Primats und Ertz-Stiffts Magdeburg/ Hertzogens zu Sachsen [...], (1652).

Krünitz J. G., Johann Georg Krünitz 'ökonomisch-technologische Encyklopädie oder allgemeines System der Staats-, Stadt-, Haus-, und Landwirtschaft und der Kunstgeschichte, 188 (1846). 
[Lehmann P. A.], Die vornehmsten Europaeischen Reisen/ Wie solche durch Deutschland, Frankreich, Italien, Holl- und Engelland, Dänemark und Schweden, Vermittelst der darzu verfertigten Reise-Carten nach den bequemsten Post-Wegen anzustellen [...], (1713).

Marperger P. J., Vollständiges Küch- und Keller-Dictionarium, (1717).

Müller J. Ch., Meines Lebens Vorfälle \& Neben-Umstände, 1: Kindheit und Studienjahre (1720-1746), (2007).

Neu-revidirte Willkühr der Stadt Danzig, aus Schluß Sämmtlicher Ordnungen, (1761)

[Schartow J. F. B.], Die Polen. Ein Beitrag zur Charakteristik dieses Volkes aus den Erinnerungen eines alten Justiz-Beamten, (1848).

Schopenhauer J., Ihr glücklichen Augen. Jugenderinnerungen. Tagebücher. Briefe, ( $\left.{ }^{2} 1979\right)$.

Serenissimi Verordnung wegen der Gasthöfe in Dero Residenz=Stadt Wolfenbüttel, (1748).

Stoud F., Das Reise-Collegium des Hofrat Schlözers gehalten im Winter 1792-1793, in: U. Kutter, Reisen - Reisehandbücher - Wissenschaft. Materialien zur Reisekultur im 18. Jahrhundert, (1996).

Uklanski C. Th., Briefe über Polen, Österreich, Sachsen, Bayern, Italien, Etrurien, den Kirchenstaat und Neapel, an die Comtesse Constance de S., 1 (1808).

Verordnung Des Hochgebornen Herrn, Herrn Heinrichen des Andern Jüngern und der Zeit Eltisten Reußen Herrn von Plauen, Herrn zu Graitz, Crannichfeld, Gerau, Schleitz und Lobenstein etc. [...], (1664).

Von Gottes Gnaden Wir Carl, Herzog zu Braunschweig und Lüneburg [...] Fügen hiemit zu wissen, (1747).

Werdum von U., Das Reisejournal des Ulrich von Werdum (1670-1677), hg. v. S. Cramer, (1990).

\section{LITERATURA / LITERATUR / LITERATURE}

Bornschein E., Geschichte von Polen vom Ursprung dieses Reichs an bis auf die neuesten Zeiten, (1808).

Chartier R., Lesewelten. Buch und Lektüre in der frühen Neuzeit, (1990).

Hahn H. H., Hahn E., Nationale Stereotype. Plädoyer für eine historische Stereotypenfoschung, in: Stereotyp, Identität und Geschichte, hg. v. H. H. Hahn, (2002), S. 17-56.

Hermeier A., Postkutschen-Passagiere als privilegierte Kaffeetrinker, in: Archiv für Postgeschichte, 1 (1982), S. 94-95.

Holste K., Das Wechselspiel von Fremd und Selbstexotisierung polnischlitauischer Eliten im letzten Drittel des 18. Jahrhunderts, in: Konstruktionen Europas in der Frühen Neuzeit. Geographische und historische Imaginationen, hg. v. S. Richter, M. Roth, S. Meurer, (2017), S. 97-115.

Kawiarnia Mombera, in: Gedanopedia, URL: https://www.gedanopedia.pl/gdansk/? title=KAWIARNIA_MOMBERA (31.05.2020).

Kizik E., Die Gäste der Festung Weichselmünde bei Danzig im 17. und 18. Jahrhundert, in: Reisen, Wahrnehmen, Kommunizieren im deutsch-polnischen Kontext in der Frühen Neuzeit. Festschrift zum 65.Geburtstag von Prof. Włodzimierz Zientara, hg. v. J. Kodzik, A. Mikołajewska, (2019), S. 71-78.

Kodzik J., Im Wirtshaus und in der Postkutsche. Herrnhutische Kommunikationsstrategien auf Reisen am Beispiel Polen-Litauens, in: Reisen, Wahrnehmen, Kommunizieren im 
deutsch-polnischen Kontext in der Frühen Neuzeit. Festschrift zum 65.Geburtstag von Prof. Włodzimierz Zientara, hg. v. J. Kodzik, A. Mikołajewska, (2019), S.79-98.

Kotarska J., „Karczem dwie krzyżowego gościńca pilnuja“. Społeczne przestrzenie staropolskiej karczmy, in: Rzeczpospolita domów, (2019), S. 82-97.

Kotarski E., Regulacje prawne osiemnastowiecznego Gdańska dotyczące gospód i kawiarni in: Rzeczpospolita domów, 5: Karczmy zajazdy, gospody, hg. v. K. Krawiec-Złotkowska, (2019), S. 15-24.

Mielnik M., Siedlisko grzechu, czyli karczmy w sztuce gdańskiej na tle sztuki europejskiej w XVI i na początku XVII wieku, in: Rzeczpospolita domów, 5: Karczmy zajazdy, gospody, hg. v. K. Krawiec-Złotkowska, (2019), S. 26-34.

Orłowski H., „Polnische Wirtschaft“. Zum deutschen Polendiskurs der Neuzeit, (1996).

Raff G. Ch., Geographie für Kinder, $1\left({ }^{4} 1787\right)$.

Schwerhoff G., Die Policey im Wirtshaus. Obrigkeitliche und gesellschaftliche Normen im öffentlichen Raum der Frühen Neuzeit. Das Beispiel der Reichsstadt Köln, in: Machträume der frühneuzeitlichen Stadt, hg. v. Ch. Hochmuth, S. Rau, (1991).

Simon-Muscheid K., Die Dinge im Schnittpunkt sozialer Beziehungsnetze: Reden und Objekte im Alltag (Oberrhein, 14. Bis 16.Jahrhundert), (2004).

Straubel R, Biographisches Handbuch der preußischen Verwaltungs- und Justizbeamten 1740-1806, Biographien, 1: A-L, 2: M-Z, (2009).

Szarota E. M., Die gelehrte Welt des 17. Jahrhunderts über Polen, (1972).

Tlusty A., Bacchus und die bürgerliche Ordnung. Die Kultur des Trinkens im frühneuzeitlichen Augsburg, 1 (Studien zur Geschichte des Bayerischen Schwaben 34, 2005).

Walaszek A., Migracje Europejczyków 1650-1914, (2007).

Wallentin S., Fürstliche Normen und akademische „Observanzen“. Die Verfassung der Universität Jena 1630-1730, (Veröffentlichungen der Historischen Kommission für Thüringen Kleine Reihe 27, 2009).

Wyżga M., Homo movens. Mobilność chłopów w mikroregionie krakowskim w XVI-XVIII wieku, (2019).

Wyżga M., Ze współczesnych badań nad mobilnością w Europie przednowoczesnej, in: Przeszłość Demograficzna, 40 (2018), S.11-37.

Zientara W., Essen unterwegs. Zwischen Angstgefühlen und Anerkennung für die Wirtshäuser auf Reisen in der Frühen Neuzeit, in: Documenta Pragensia, 25 (2007), S. 241-250.

Zientara W., Niemieckie media XVII wieku o Rzeczpospolitej szlacheckiej, in: Z szabla i kielichem, czyli życie po sarmacku, hg. v. J. Tazbir, S. Zagórski, (2015), S. 183-194.

Zientara W., Reise nach Danzig Anfang des 19. Jahrhunderts, in: Hybride Identitäten in den preußisch-polnischen Stadtkulturen der Aufklärung. Studien zur Aufklärungsdiffusion zwischen Stadt und Land, zur Identitätsbildung und zum Kulturaustausch in regionalen und internationalen Kommunikationsnetzwerken, hg. v. J. Kodzik, W. Zientara, (2016), S. 261-274.

Zientara W., Sarmatia Europiana oder Sarmatia Asiana? Polen in den deutschsprachigen Druckwerken des 17. Jahrhunderts, (22003).

Zwischen Gotteshaus und Taverne. Öffentliche Räume in Spätmittelalter und Früher Neuzeit, hg. v. S. Rau, G. Schwerhoff, (2004). 